\title{
OSG-057 Superconducting Gravimeter Noise Levels in Lhasa (China)
}

\author{
Miaomiao Zhang ${ }^{1,2, *}$, Jianqiao $\mathrm{Xu}^{1}$, Heping $\mathrm{Sun}^{1}$, Xiaodong $\mathrm{Chen}^{1}$, and Jiangcun Zhou ${ }^{1}$ \\ ${ }^{1}$ State Key Laboratory of Geodesy and Earth's Dynamics, Institute of Geodesy and Geophysics, Chinese Academy of Sciences, \\ Wuhan, China \\ ${ }^{2}$ University of Chinese Academy of Sciences, Beijing, China
}

Received 7 August 2015, revised 7 March 2016, accepted 23 March 2016

\begin{abstract}
This research tests OSG-057 superconducting gravimeter (SG) performance in Lhasa in terms of noise levels in the seismic ( 2 min to $1 \mathrm{~h}$ ), sub-seismic (1 to $6 \mathrm{~h}$ ), and tidal (above $6 \mathrm{~h}$ ) bands. Based on the Seismic Noise Magnitude (SNM) and Sub-Seismic Noise Magnitude (SSNM), 0.867 and 2.608 respectively, and compared to the noise levels of other SGs in the Global Geodynamics Project (GGP)/International Geodynamics and Earth Tide Service (IGETS), it is concluded that the instrument-site combination in Lhasa has low noise and good quality in both the seismic and sub-seismic bands. The seismic and sub-seismic band results were confirmed by large signal-to-noise ratio amplitude spectra observations below 1 milliHertz (mHz) obtained from OSG-057 data record residuals after the 4/11/2012 M8.6 Sumatra earthquake, and by background free Earth oscillation observations ('hum'), although very weak. Using the ETERNA Earth Tide Analysis program in the tidal band, it was found that the OSG-057 performs well due to the low average noise amplitudes and small standard deviations. Knowledge of the noise levels of the OSG-057 SG in Lhasa in different frequency bands provides a necessary precondition and reference for its applications to both global and regional geodynamical researches.
\end{abstract}

Key words: Noise levels, OSG-057 superconducting gravimeter, Lhasa

Citation: Zhang, M., J. Xu, H. Sun, X. Chen, and J. Zhou, 2016: OSG-057 superconducting gravimeter noise levels in Lhasa (China). Terr. Atmos. Ocean. Sci., 27, 807-817, doi: 10.3319/TAO.2016.03.23.01(T)

\section{INTRODUCTION}

With extremely high sensitivity, long-term stability, wide dynamic linearity measurement range and low noise level (Sun et al. 1999, 2001), superconducting gravimeters (SGs) are known to be the most precise and stable relative gravity meters in existence (Goodkind 1991). SGs are regarded as an important tool in geophysics, geodynamics and geodesy. Due to its advantages an international network of SGs-Global Geodynamics Project (GGP), endorsed by the International Union of Geodesy and Geophysics (IUGG) inter-union project Study of the Earth's Deep Interior (SEDI), was established in 1997 for monitoring changes in the Earth's gravity field from seconds (e.g., seismic normal modes) to years (e.g., tectonic deformation) (Crossley et al. 1999). In 2015, the GGP reached the end of its working lifetime and became a service of the International Association of Geodesy (IAG) and of the Global Geodetic Observing System

\footnotetext{
* Corresponding author

E-mail:zhangmm@whigg.ac.cn
}

(GGOS), entitled the International Geodynamics and Earth Tide Service (IGETS), continuing GGP activities to provide support to geodetic and geophysical research activities using SG data within the context of an international network. At present, this SG network includes more than 30 operating stations distributed worldwide. This network has provided data for a number of years for gravity change studies associated with local and global phenomena (e.g., tidal variations, ocean loading, atmospheric loading, hydrology, polar motion, the nearly diurnal resonance effect of the free core nutation, and the search for the Slichter triplet and core modes) (Hinderer and Crossley 2000; Meurers 2001; Crossley et al. 2005; Crossley and Hinderer 2009). Lhasa is situated on the Central Tibetan Plateau and north of the Himalayas, in the middle reaches of the Lhasa River valley plain, a tributary of the Yarlung Zangbo River. On December 2009, the OSG-057 SG (cf. Fig. 1) was installed in Lhasa by the Institute of Geodesy and Geophysics, Chinese Academy of Sciences. Since then the Lhasa SG has been collecting gravity observations and playing a very important role in both global and regional 
research projects, such as the GGP/IGETS, the Asia-Pacific Space Geodynamics Project (APSG) and the Crustal Movement Observation Network of China (CMONOC), contributing to the study of Earth tides, the nearly diurnal-free wobble and modes of the Earth's core, Earth's rotation and polar motion, Earth interactions with the atmosphere and oceans, gravity changes due to tectonic motions, regional seasonal and seismic effects, seismic modes and so on (Sun and Xu 1997; Crossley et al. 1999; Courtier et al. 2000). However, there are only a few detailed studies on the performance of the OSG-057 in different frequency bands. This research tests the OSG-057 performance in terms of noise levels in the seismic ( 2 min to $1 \mathrm{~h}$ ), sub-seismic (1 to $6 \mathrm{~h}$ ), and tidal (above $6 \mathrm{~h}$ ) bands, using data spanning from 1 January 2010 to 31 December 2013.

\section{NOISE LEVELS IN THE SEISMIC (2 MIN TO 1 H) AND SUB-SEISMIC (1 TO 6 H) BANDS}

\subsection{The Method and Results}

Here we first compute the OSG-057 noise level in the seismic band with the processing procedure proposed by Banka and Crossley (Banka 1997; Banka and Crossley 1999) and recommended by the GGP/IGETS (http://www.eas.slu. edu/GGP/ggphome.html). The Seismic Noise Magnitude (SNM) concept, similar to the earthquake magnitude, is introduced in this procedure to quantify and compare the noise levels at seismic frequencies. Following nearly the same procedure, the OSG-057 noise level in the sub-seismic band is computed with respect to the term Sub-Seismic Noise Magnitude (SSNM) (Rosat et al. 2003, 2004; Rosat and Hinderer 2011) generalized from SNM. Based on this method, Rosat et al. $(2003,2004)$ enabled the quantitative comparison of SG noise levels at the GGP/IGETS stations in these two bands. Knowledge of the noise levels at each station in the seismic and sub-seismic bands is important for site selection, instrumental modifications, evaluation of the recent potential of SGs in seismic normal mode studies and in the search for the Slichter mode, and combination of the SGs to determine global Earth parameters (Banka 1997; Rosat et al. 2003, 2004). The processing procedure for studying the noise level in the seismic band is described briefly as follows.

On a yearly basis the one-minute interval raw gravity and pressure daily files of the OSG-057 were assembled and calibrated in amplitude from volts to microgal ( $\mu \mathrm{gal})$ and to hectopascal $(\mathrm{hPa})$, respectively. The pressure files were fixed for spikes, gaps, and offsets to avoid transferring problems in the pressure into the gravity data. A synthetic elastic tide, based on a modern tidal potential (Tamura 1987; Xi 1989) or later with recent values for the elastic tidal Love numbers was subtracted and the influence of the air pressure was reduced with an admittance factor of $-0.3 \mu \mathrm{gal} \mathrm{hPa}^{-1}$. In order to eliminate the instrument drift and any residual tidal signal, a best-fitting $9^{\text {th }}$ degree polynomial was subtracted.
The daily Root-mean-square (RMS) of the reduced gravity data was then computed and the 5 quietest days with the lowest RMS were selected. A Fast Fourier Transform (FFT) for the data from each of the 5 quietest days was taken through windowing with the Hann window and the data was padded with zeros to the $(n e x t+1)$ power of 2 . The average of the 5 unnormalized amplitude spectra was then computed. According to the average FFT spectrum the Power Spectral Densities (PSD) were plotted and the mean PSD [unit: microgal $^{2}$ milliHertz $^{-1}\left(\mu \mathrm{gal}^{2} \mathrm{mHz}^{-1}\right)$ ] was computed in the period range $200-600 \mathrm{~s}$ to acquire the SNM through the relation (Banka 1997):

$\mathrm{SNM}=\log _{10}($ mean PSD $)+5.5$

The OSG-057 SNMs are listed in Table 1, from which we can see that the SNMs are 0.9 or so and did not change much from 2010 - 2013, i.e., noise levels in the seismic band are generally stable during $2010-2013$. The SNM is 0.867 for the whole period.

The PSDs of the OSG-057 are shown in Fig. 2, referring to the New Low Noise Model (NLNM) (Peterson 1993), which is a reference noise model in seismology and represents the lower bound for the best seismometers. As a matter of fact, removing a $9^{\text {th }}$ degree polynomial artificially decreases the PSDs at low frequencies, resulting in the lower PSD curve of the SG with respect to that of the seismometer. However, in the 200 - 600 s period range the PSD curve of the SG is always higher than that for the seismometer. Because the SNM $(0.867$, below 1.0) is low compared to the noise levels of other SGs in the GGP/IGETS [cf. Fig. 2 of Rosat et al. (2004)], it is affirmed that the instrument-site combination (noises at a station come from both the instrument and site) in Lhasa has low noises and good quality in the seismic band.

Slightly different from the above mentioned processing procedure, the $9^{\text {th }}$ degree polynomial was replaced by high-pass filtering with a corner period of $8 \mathrm{~h}$. Instead of computing the RMS for each day and selecting the 5 quietest days with the lowest RMS, we computed the RMS with a moving window of 15 days shifted by 1 day and then chose the quietest 15 continuous days with the lowest RMS (Rosat et al. 2004). In addition, the PSD was smoothed in the frequency domain with a 101-point moving average. The SSNMs of the OSG-057 are listed in Table 2, from which we find that the SSNMs change slightly from 2010 - 2013, i.e., noise levels in the sub-seismic band are almost stable during 2010 - 2013. For the whole period, the SSNM is 2.608.

The PSDs of the OSG-057 are shown in Fig. 3, also referring to the NLNM (Peterson 1993). Compared to the noise levels of other SGs in the GGP/IGETS [cf. Fig. 5 of Rosat et al. (2004)], it is concluded that the instrument-site combination in Lhasa is not noisy in the sub-seismic band in 
terms of the small SSNM (2.608). In fact, the results in this study are consistent with the conclusion that high linear correlation exists between the noise levels in the seismic and sub-seismic frequency bands, and thus estimating the noise level in only the seismic frequency band would be sufficient (Rosat et al. 2004; Rosat and Hinderer 2011).

\subsection{Some Proofs}

Furthermore, on the one hand, the above results were confirmed and the better quality of the OSG-057 data in the long-period seismic band underlined, owing to the observations of its larger signal-to-noise ratio amplitude spectra versus that of the STS-1/Z data below $1 \mathrm{mHz}$, where the Eigen frequencies of the low-order free oscillation modes (which are not excited to large amplitudes even by very large earthquakes) are located (Richter et al. 1995; Freybourger et al. 1997). Herein the STS-1/Z is the only available seismometer in Lhasa for the study of free oscillation modes excited by earthquakes and is about $11 \mathrm{~km}$ away from the OSG-057

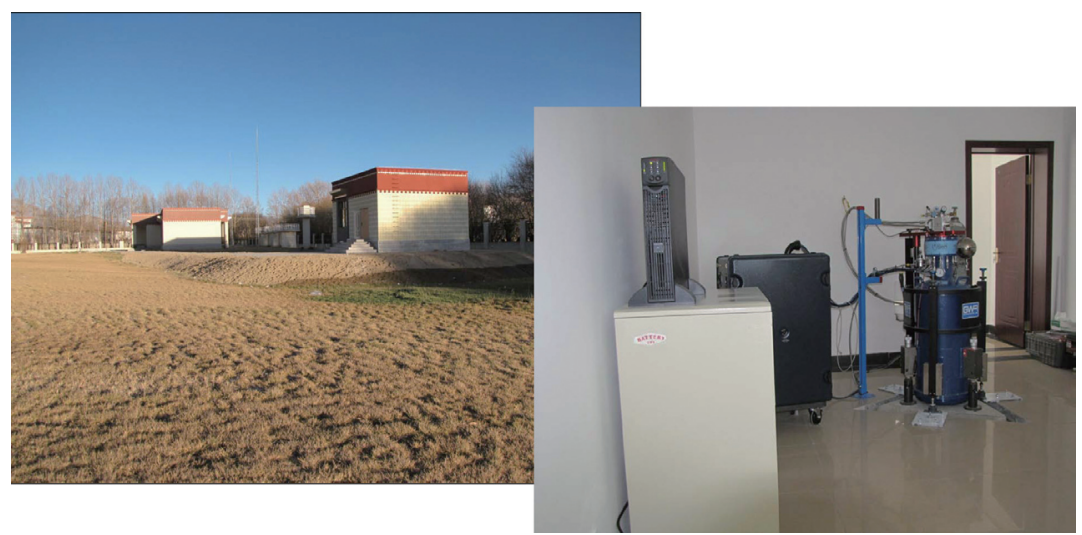

Fig. 1. OSG-057 superconducting gravimeter $\left(29.645^{\circ} \mathrm{N}, 91.035^{\circ} \mathrm{E}\right)$ operating in the urban area of Lhasa, China.

Table 1. SNMs of the OSG-057 from 2010 - 2013.

\begin{tabular}{ccc}
\hline Year & The $\mathbf{5}$ quietest days & SNM \\
\hline 2010 & $21,26,32,6,171$ & 0.885 \\
2011 & $34,365,322,50,324$ & 0.892 \\
2012 & $49,6,13,50,322$ & 0.909 \\
2013 & $50,64,27,68,36$ & 0.950 \\
\hline All years & $21(2010), 34(2011), 26(2010), 32(2010), 365(2011)$ & 0.867 \\
\hline
\end{tabular}

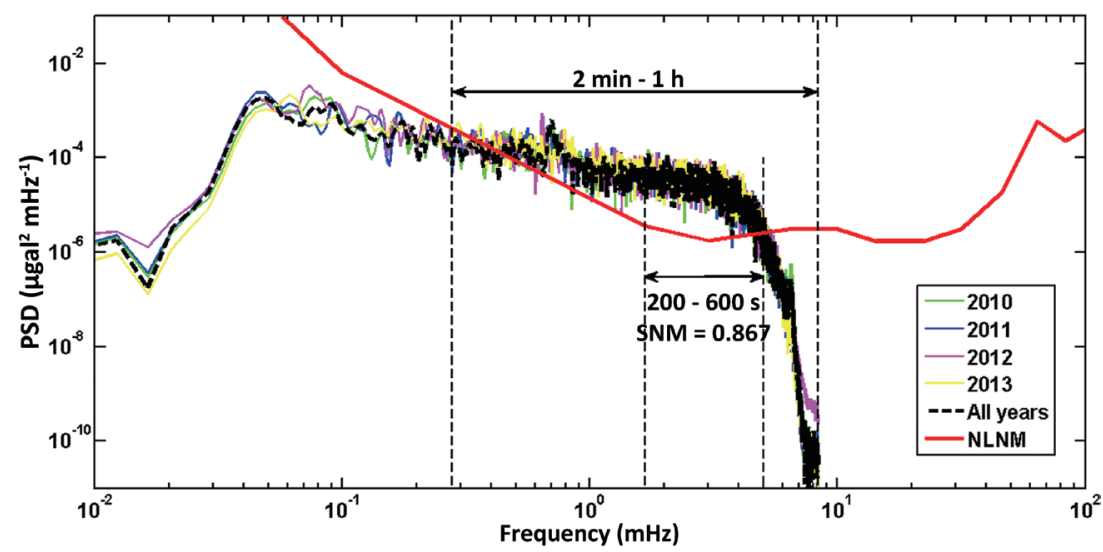

Fig. 2. Power spectral densities of the 5 quietest days of each year between 2010 and 2013, and of all years at Lhasa SG station. The noise level in the seismic band for all four years is quantified by the Seismic Noise Magnitude (SNM = 0.867) computed in the $200-600 \mathrm{~s}$ band. The New Low Noise Model (NLNM) is used as a reference for the noise levels. 
Table 2. SSNMs of the OSG-057 from 2010 - 2013.

\begin{tabular}{ccc}
\hline Year & The quietest 15 continuous days & SSNM \\
\hline 2010 & $25-39$ & 2.608 \\
2011 & $270-284$ & 2.829 \\
2012 & $13-27$ & 2.814 \\
2013 & $22-36$ & 2.645 \\
\hline All years & $25-39(2010)$ & 2.608 \\
\hline
\end{tabular}

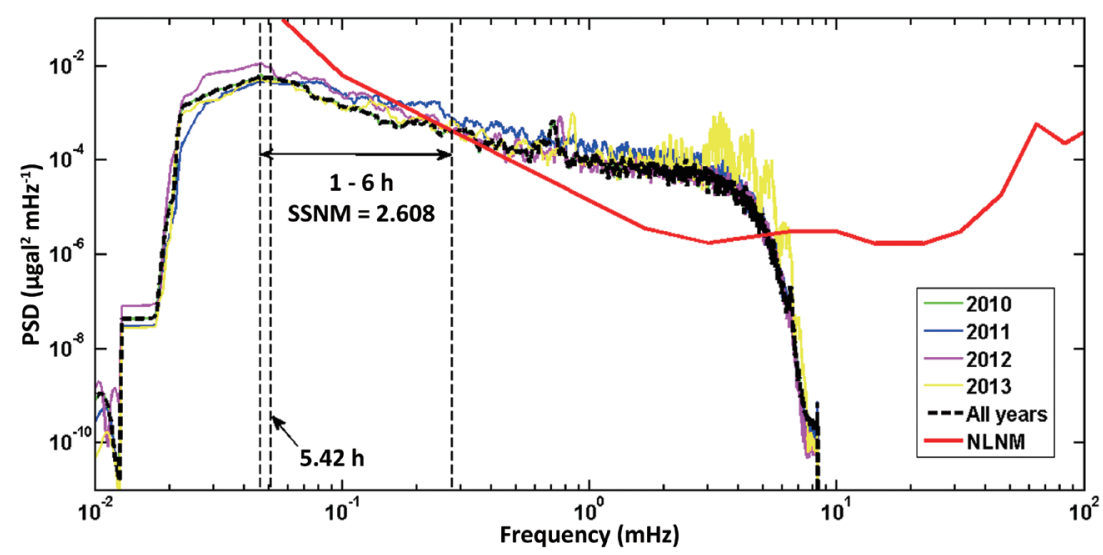

Fig. 3. Power spectral densities of the quietest 15 continuous days of each year between 2010 and 2013, and for all years at Lhasa SG station. The noise level in the sub-seismic band for all four years is quantified by the Sub-Seismic Noise Magnitude (SSNM = 2.608) computed in the band 1 - 6 h. The New Low Noise Model (NLNM) is also used as a reference for the noise levels. The theoretical central period $5.42 \mathrm{~h}$ of the Slichter triplet from Earth Model PREM (Dziewonski and Anderson 1981) is also marked.

(cf. Fig. 4). Given the wavelengths of the gravity signals we are interested in (e.g., about $20000 \mathrm{~km}$ for the fundamental mode ${ }_{0} \mathrm{~S}_{2}$ ), the SG and STS-1/Z stations are actually close enough to make the observed signals similar and comparable in both waveforms and amplitudes (Freybourger et al. 1997). Both the SG and STS-1/Z data records were one-second decimated and the STS-1/Z data records were corrected for the instrumental response to recover ground acceleration. Figure 5 shows the time fluctuations of gravity signals for a 5 - 48 h time window after the 4/11/2012 M8.6 Sumatra earthquake recorded by the SG and STS-1/Z, respectively. In general, they produced consistent results. Figure 6 gives the amplitude spectra of the residuals (with the local tides and the barometric pressure effect subtracted using tidal parameters and the barometric pressure admittance derived from tidal analysis). Clearly, due to the lower instrumentsite combination noise level, the OSG-057 performs better and has much larger SNRs for all excited peaks in this band, including fundamental toroidal mode ${ }_{0} \mathrm{~T}_{2}$ (which is observed by SG due to mode-coupling through rotation or 3-dimensional structure), fundamental spheroidal modes ${ }_{0} \mathrm{~S}_{3},{ }_{0} \mathrm{~S}_{4},{ }_{0} \mathrm{~S}_{5}$, and spheroidal overtone ${ }_{1} S_{2}$. Additionally, considering that the STS- $1 / \mathrm{Z}$ is generally operated under good conditions, it is assumed that the high noise level of this seismic station is most probably attributed to the instrument itself rather than site effects. As has been proven by Rosat et al. (2003), the new generation of SGs could outperform the best seismometers in the long-period seismic band.

On the other hand, the background free oscillations of the Earth, which are commonly called 'hum' and provide a good reference for the noise level evaluation in the $\mathrm{mHz}$ band (Nawa et al. 2000), are observed in the OSG-057 data records and thus indicate that the Lhasa SG station has low noises in the seismic and even sub-seismic bands. Following the method recommended by Nawa et al. (2000), both the local synthetic tides and the pressure effect with an admittance of about $-0.3 \mu \mathrm{gal} \mathrm{hPa}^{-1}$ were removed from the one-minute interval data (the glitches and offsets were also corrected). The power spectra for every seismically quiet period, which is defined as a 3-day-long interval not containing the day of or day immediately after any earthquakes with moment magnitude greater than 5.7 listed in the Global CentroidMoment-Tensor (CMT) catalogues, were computed. These power spectra were then stacked and further smoothed with an 11-point moving average to obtain the averaged power spectrum. Given that the background free oscillations were detected early in the 1990s at Syowa (Antarctica, T016) and Canberra (Australia, C031) SG stations, these two stations were taken as a reference. Figure 7 shows the averaged power spectra between 0.3 and $5 \mathrm{mHz}$ during the year 2010 for 


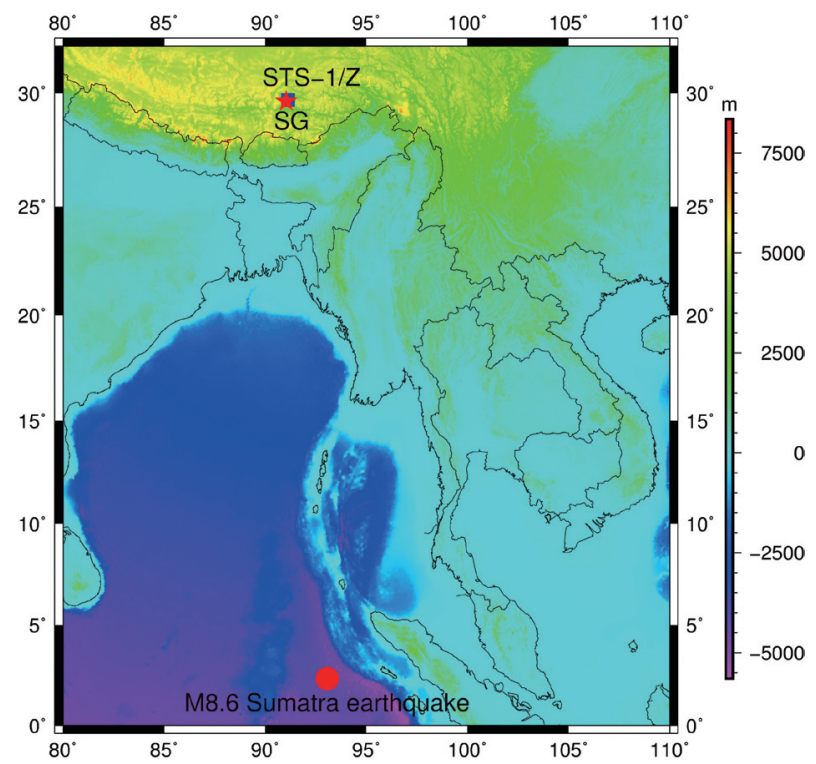

Fig. 4. Locations of the OSG- $057\left(29.645^{\circ} \mathrm{N}, 91.035^{\circ} \mathrm{E}\right.$; red star $)$, the STS- $1 / \mathrm{Z}\left(29.703^{\circ} \mathrm{N}, 91.127^{\circ} \mathrm{E}\right.$; blue square $)$, and the epicenter of the $4 / 11 / 2012$ M8.6 Sumatra earthquake $\left(2.327^{\circ} \mathrm{N}, 93.063^{\circ} \mathrm{E}\right.$; red circle).

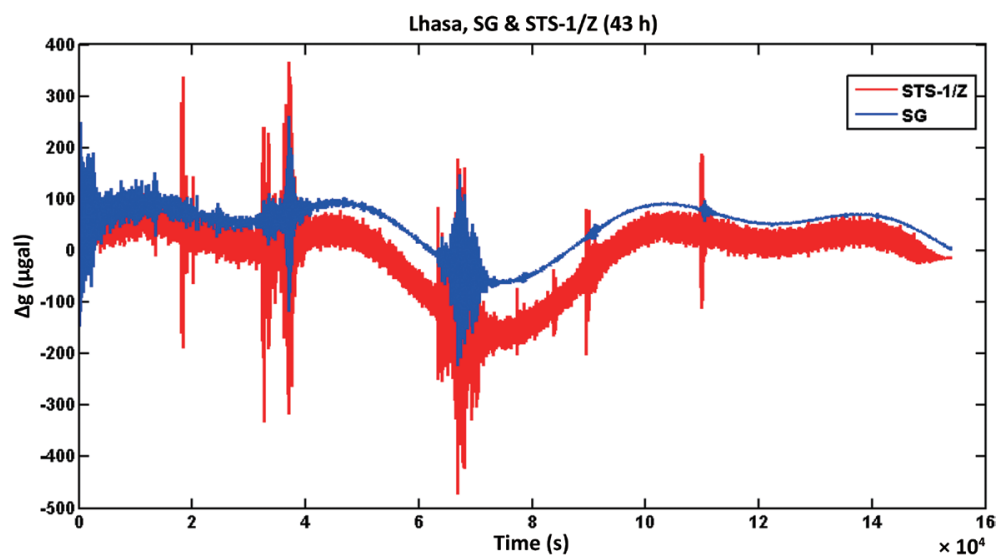

Fig. 5. Time fluctuations of gravity signals for time window from 5 - $48 \mathrm{~h}$ after the 4/11/2012 M8.6 Sumatra earthquake recorded by the SG and STS-1/Z respectively.

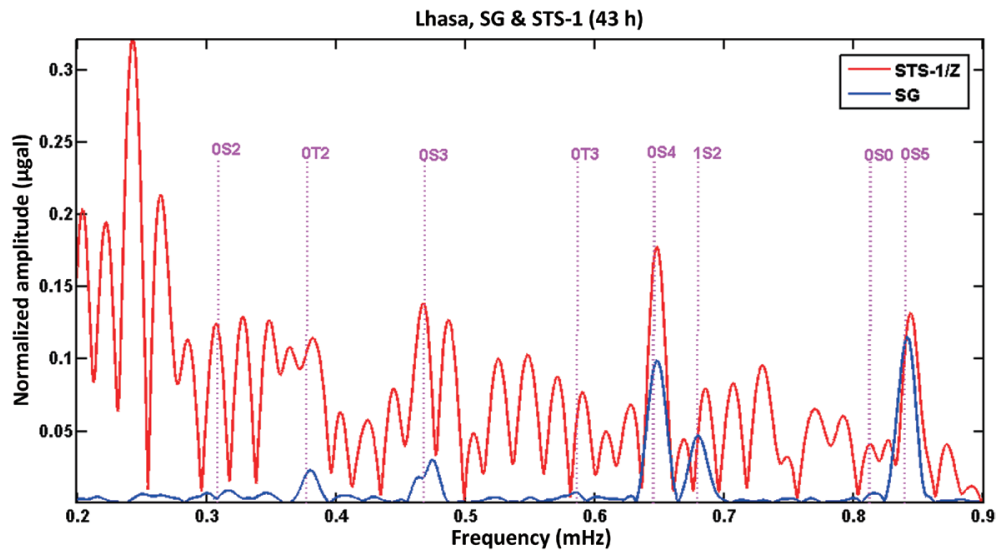

Fig. 6. Amplitude spectra obtained from the residuals of the SG and STS-1/Z respectively. Vertical magenta dotted lines indicate theoretical Eigen frequencies of some free oscillation modes from Earth Model PREM (Dziewonski and Anderson 1981). 


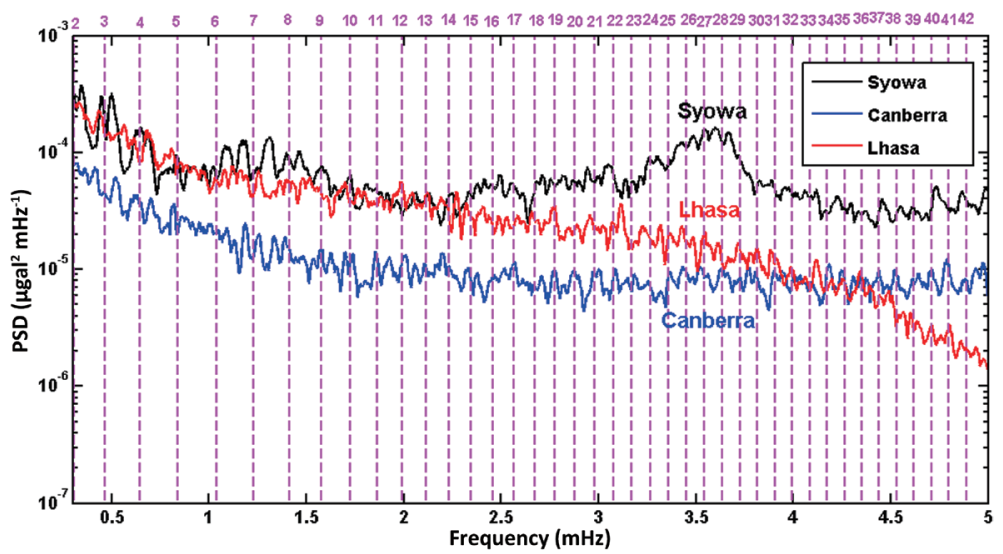

Fig. 7. Averaged power spectra between 0.3 and $5 \mathrm{mHz}$ (with logarithmic scale for Y axis) for seismically quiet periods with a cut-off magnitude of 5.7 in 2010 for Lhasa (OSG-057), Canberra (C031), and in 1998 for Syowa (T016). Vertical magenta dashed lines indicate theoretical Eigen frequencies of some fundamental spheroidal modes from Earth Model PREM (Dziewonski and Anderson 1981), and numbers at the top frame are angular orders of these modes.

Lhasa and Canberra and the year 1998 for Syowa (stopped on January 2003). The spectral peaks of the background free oscillations are most clear at Canberra station, then at Lhasa station and finally at Syowa station [high local noise level at Syowa station probably due to oceanic effects as mentioned by Nawa et al. (2000)], which is consistent with the noise levels comparison, i.e., the SNM (Canberra) $<$ SNM (Lhasa) $<$ SNM (Syowa). Our results are consistent with the critical noise level, $10^{-17} \mathrm{~m}^{2} \mathrm{~s}^{-3}$ or $10^{-4} \mathrm{\mu gal}^{2} \mathrm{mHz}^{-1}$ (Nawa et al. 2000), below which the background free oscillations can be easily identified. Additionally, more intuitive background free oscillation observations were acquired through the following procedure: the residuals (with the local tides and the barometric pressure effect subtracted) were divided into 3 -day-long records with a time lag of 1 day; each record was tapered with a normalized Hanning taper and then Fouriertransformed to obtain its power spectrum; the successive power spectra were pasted up along a time axis to obtain a spectrogram (Nawa et al. 2000). Figures 8a and b show almost the same background free oscillations (i.e., weak, continuous vertical lines) distribution characteristics as Nawa et al. (1998, 2000): at Syowa station there are high amplitudes at frequencies less than $2 \mathrm{mHz}$, nearly 2.5 and $3-4 \mathrm{mHz}$; at Canberra station the spectral level at frequencies $2-5 \mathrm{mHz}$ is almost flat both in time and frequency. Figure $8 \mathrm{c}$ also shows the flat spectral level of the background free oscillations at frequencies $2-4.5 \mathrm{mHz}$ at Lhasa station, although weak and less clear. Here it is worth mentioning that the appearance of the distinct vertical line around $5.5 \mathrm{mHz}$ in Fig. $8 \mathrm{a}$ is due to the sphere resonance of T016 at Syowa station rather than representing the background free oscillations. Because major earthquakes have been taken out in the pre-processing step, the horizontal lines corresponding to the excited normal modes are not very obvious in Fig. 8. The spectral level of the background free oscillations at about $10^{-18}-10^{-17} \mathrm{~m}^{2} \mathrm{~s}^{-3}$ (a little higher for Syowa) in Fig. 8 is roughly comparable to that of Nawa et al. (2000) (while the discrepancies between them are related to different sample rates and intervals as well as the different corrections for glitches and offsets applied). It is deduced that the distribution characteristics of the background free oscillations can be quite different at different stations, although excited by global and random sources in a thin layer just above the Earth's surface (Nishida et al. 2002). It is guessed that the background free oscillations may be stable during the 1990s - 2010 period at least for Canberra station. Further analyses of more data records at quiet SG stations are already under way by more advanced signal processing methods.

\section{NOISE LEVEL IN THE TIDAL BAND (ABOVE 6 H)}

The noise level estimation for the OSG-057 in the tidal band is carried out in this part using the ETERNA Earth Tide Analysis program (Wenzel 1996). After numerical filtering and decimation from $1 \mathrm{~s}$ to $1 \mathrm{~min}$ the data were pre-processed for gaps, spikes, steps and other disturbances with the TSoft software (Van Camp and Vauterin 2005), and then decimated to the hourly data. By applying a Tschebyscheff polynomial, the tidal potential development of Tamura (1987) and a linear regression with air pressure, ETERNA (Wenzel 1996) performed the tidal analysis on the hourly data spanning from 1 January 2010 to 31 December 2013. The noise levels estimated from the Fourier spectral analysis of the OSG-057 residual records at different tidal frequencies are listed in Table $3\left[0.1,1,2,3\right.$, and 4 cycles day $^{-1}(\mathrm{cpd})$ represent long-periodic (periods between 0.007 and 0.193 cpd), diurnal, semidiurnal, terdiurnal, and quaterdiurnal tidal waves, respectively]. The standard deviations after highpass filtering were also computed $\left\{0.437 \mathrm{~nm} \mathrm{~s}^{-2}\right.$ for 2010 , 
$0.429 \mathrm{~nm} \mathrm{~s}^{-2}$ for $2011,0.469 \mathrm{~nm} \mathrm{~s}^{-2}$ for 2012, $0.440 \mathrm{~nm} \mathrm{~s}^{-2}$ for 2013, and $2.342 \mathrm{~nm} \mathrm{~s}^{-2}$ for 2010 - 2013 [longer series often have higher standard deviation (Ducarme and Vandercoilden 2000)]\}. These results indicate the low noise level at Lhasa station referring to those at other quiet SG stations [cf. Table 1 of Freybourger et al. (1997) and Table 1 of Rosat et al. (2004)].

The amplitude factors $\delta$ for the four main tidal waves $\left(\mathrm{O}_{1}, \mathrm{~K}_{1}, \mathrm{M}_{2}, \mathrm{~S}_{2}\right)$ from this study, together with those from Xu et al. (2012) (who used only 447 days of data) and those from the theoretical model DDW99 (Dehant et al. 1999), are shown in Table 4. It is found that our results, though not corrected for ocean loading effect, in general agree with the results in Xu et al. (2012) (corrected for ocean loading effect) and even match better with those given by the DDW99. The discrepancies between our results and those given by the DDW99 are likely to be related to the active Tibetan Plateau geodynamic activity and very thick crust (i.e., deep Moho depth) in the local region, and can be further decreased by removing both ocean loading and local pressure effects $(\mathrm{Xu}$ et al. 2012). Additionally, the internal precision of the tidal analysis results obtained (i.e., RMS errors of the amplitude factors) is higher, i.e., 0.13, 0.10, 0.07, 0.15 for the $\mathrm{O}_{1}, \mathrm{~K}_{1}$, $\mathrm{M}_{2}$, and $\mathrm{S}_{2}$ waves, respectively, which is a consequence of the small residual noise levels in Table 3 (Freybourger et al. 1997). As a result, our results can sufficiently be regarded as local tidal gravity references for the gravity measurements in the Tibetan plateau and its adjacent areas, and the Lhasa SG station can be collaborated with other SG stations for the studies of Earth tides, the validation of solid Earth and ocean tidal models (Baker and Bos 2003; Boy et al. 2003) and so on.

\section{DISCUSSION}

Although the SNMs listed in Table 1 are generally small (below 1), it seems that the SNM increases from 2010 - 2013, especially in 2012 and 2013 . One possible reason is the instrument itself, considering a similar case in Zhang et al. (2014) where the higher noise level of the new type superconducting gravity meter (iGrav-007) in the seismic band can be attributed to the SG's Dewar operating conditions. However, the specific causes need further investigation.

Figure 3 marks the theoretical central period (5.42 h) of the Slichter triplet from the Earth Model PREM (Dziewonski and Anderson 1981), the detection of which, though claimed by Smylie (1992), has been controversial. Although much effort such as Hinderer et al. (1995), Rosat et al. (2003, 2004), and Ding and Chao (2015) has been made to observe the Slichter triplet through different methods and SG data, no significant signals corresponding to those reported by Smylie (1992) or those predicted by theoretical Earth models have been found. In the next years, the Slichter triplet may still elude observation unless there would be larger earthquakes and more good quality SG data (communication with Ding within the $6^{\text {th }}$ TibXS Workshop, 2015). Given its low noise level in the sub-seismic band and its geographical location, there is a chance for us to combine the Lhasa SG station together with other SG stations to try to detect the Slichter triplet in future.

As mentioned in section 3, certain discrepancies exist between the observed amplitude factors and those from the theoretical model. Our speculation that this inconformity is probably attributed to the active geodynamic activity of the Tibetan plateau and very thick crust (i.e., deep Moho depth) in the local region is reasonable, considering abundant results from geological and geodetic studies about the uplift and crust thickening (i.e., Moho deepening) of the Tibetan plateau. By making use of gravity measurements at Lhasa Absolute Gravity (AG) station (near the Lhasa SG station) together with gravity measurements at the other two AG stations in southeastern Tibet and Global Positioning System (GPS) measurements at the three stations, for the first time Sun et al. (2009) quantitatively proved the uplift and crust thickening of the Tibetan plateau and the resulting mass loss and decrease of gravity beneath it. The mean observed gravity changes at Lhasa AG station during the early 1990s - early 2000s had a negative trend $\left(-1.97 \pm 0.66 \mu{\left.\mathrm{gal} \mathrm{yr}^{-1}\right)}^{-1}\right.$ [cf. Fig. 2 (left) of Sun et al. (2009)] therein. Given the good performance of SG itself in measuring long-period continuous variations of gravity, the better accuracy of SG compared to that of $\mathrm{AG}$ (about 0.1 and $1 \mu$ gal respectively) (Crossley and Hinderer 2009) and the low noise level of the Lhasa SG station at all frequencies (the low noise level at longer periods is also indicated in Figs. 2 and 3, where the PSD curves are below the NLNM curve), there is a potential for us to combine the observed gravity changes at Lhasa SG station with the observed gravity changes at Lhasa AG station and GPS data and even Gravity Recovery And Climate Experiment (GRACE) data (Sun et al. 2011) to provide a more convincing evidence of the evolution of the Tibetan plateau uplift, which in turn will help to refine the theoretical model. In addition, on the one hand, occasional offsets creeping into the AG measurements can be checked by the $\mathrm{SG}$ and thus the AG measurements can be verified to 1 - $2 \mu$ gal (the anticipated and oft-quoted accuracy) under controlled conditions (Okubo et al. 1997; Crossley and Hinderer 2009), which will allow more accurate AG data to be combined with GPS data to determine the gravity change rate and the crust thickening rate of Lhasa. On the other hand, as Crossley and Hinderer (2009) concluded, hydrological variations are normally the largest unmodelled effect in AG measurements and they also showed some examples in which combined SG and AG measurements through verifying each other can track the hydrological variations and estimate the gravity changes contributed by them (Amalvict et al. 2006; Longuevergne et al. 2007). It is therefore advisable when performing gravity measurements at Lhasa AG 

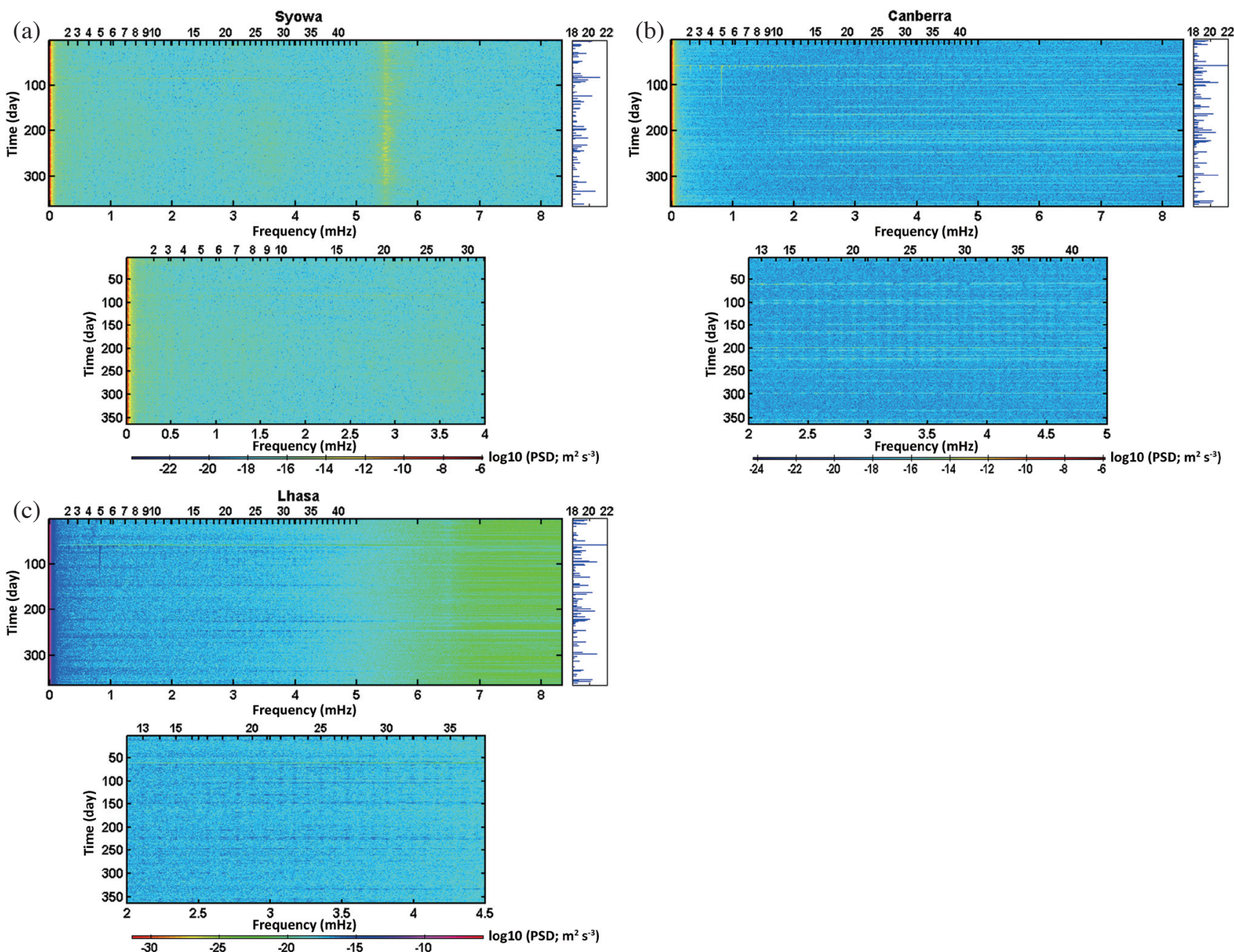

Fig. 8. Frequency-time spectrograms at (a) Syowa SG station for 1998, (b) Canberra SG station for 2010, and (c) Lhasa SG station for 2010. In each of (a), (b), and (c), the upper panel shows the whole frequency-time spectrogram between $0 \mathrm{mHz}$ and Nyquist frequency $(8.3 \mathrm{mHz})$; the lower panel shows an enlargement of the upper panel between 0 and $4 \mathrm{mHz}$ for Syowa SG station, between 2 and $5 \mathrm{mHz}$ for Canberra SG station, and between 2 and $4.5 \mathrm{mHz}$ for Lhasa SG station respectively. In each panel, numbers at the top frame are angular orders of the fundamental spheroidal modes, and bars at the right frame indicate logarithm of seismic moment $(\mathrm{Nm})$ of the Global Centroid-Moment-Tensor (CMT) solutions. The Earth's background free oscillations are expressed as weak, continuous vertical lines.

Table 3. Estimates of noise levels in different tidal frequency bands.

\begin{tabular}{cc}
\hline Frequency band $\left(\right.$ cycles day $\left.^{-1}\right)$ & Noise level of OSG-057 record $\left(\mathbf{n m ~ s}^{-2}\right)$ \\
\hline 0.1 & 0.249024 \\
1 & 0.043853 \\
2 & 0.048901 \\
3 & 0.007124 \\
4 & 0.004610 \\
\hline
\end{tabular}

Table 4. Comparison of amplitude factors $\delta$ for the four main tidal waves.

\begin{tabular}{cccc}
\hline Wave group & This paper $(\boldsymbol{\delta} \&$ RMS error $)$ & Xu et al. $(\mathbf{2 0 1 2})(\boldsymbol{\delta})$ & DDW99 $(\boldsymbol{\delta})$ \\
\hline $\mathrm{O}_{1}$ & $1.16303( \pm 0.00013)$ & 1.16601 & 1.1556 \\
$\mathrm{~K}_{1}$ & $1.13742( \pm 0.00010)$ & 1.14728 & 1.1346 \\
$\mathrm{M}_{2}$ & $1.16515( \pm 0.00007)$ & 1.17158 & 1.1594 \\
$\mathrm{~S}_{2}$ & $1.15923( \pm 0.00015)$ & 1.17016 & 1.1594 \\
\hline
\end{tabular}


station, to conduct collocated SG measurements to estimate the contribution of hydrology to gravity and thus distinguish the hydrology effects from those tectonically related. This will add useful information to the interpretation of uplift mechanisms in the Tibetan plateau since the uplift and the resulting temporal gravity changes over the Himalaya-Tibet can be generated from different mass transfer mechanisms: the glacial isostatic response induced by hydrologic and icemass changes, and the tectonic effects (Braitenberg 2014). Today, however, two main difficulties still exist: one is the limited duration of the Lhasa SG data (only about 5 years) compared to more than a decade in Sun et al. (2009); the other is the SG data preprocessing including removing offsets, eliminating the instrument drift and correcting atmospheric and oceanic loading. Therefore, some of the proposed studies in the discussion can be investigated using a longer (> one decade) SG data record at Lhasa station.

What's more, Neumeyer et al. (2005) inferred that the SG gravity variations (point measurements), if the local gravity effects are carefully removed, are representative for a large area within the $\mu$ gal accuracy. Neumeyer et al. (2006, 2008), through further temporal gravity variation comparisons resulting from SG recordings (at selected European sites or global sites in the GGP/IGETS), GRACE satellite observations and global hydrology models, showed quite a good agreement among SG, GRACE and global hydrology model-derived gravity variations. Therefore, it is possible to use gravity measurements at the low-noise Lhasa SG station like those SG stations in Neumeyer et al. $(2006,2008)$ for GRACE and global hydrology model-derived gravity variation validation. Nevertheless, the local hydrological gravity effects at Lhasa SG station should be well modelled in advance (Neumeyer et al. 2006, 2008).

\section{CONCLUSIONS}

We investigated OSG-057 SG performance in Lhasa for the first time in terms of noise levels in the seismic ( $2 \mathrm{~min}$ to $1 \mathrm{~h}$ ), sub-seismic (1 to $6 \mathrm{~h}$ ) and tidal (above $6 \mathrm{~h}$ ) bands.

Based on the small SNM and SSNM values and confirmed by the larger signal-to-noise ratio amplitude spectra observations in the long-period seismic band after a large earthquake and by weak background free Earth oscillation observations, it is affirmed that the instrument-site combination in Lhasa has low noise level and performs well in both seismic and sub-seismic bands. Based on the low average noise amplitudes and the small standard deviations, it was found that the instrument-site combination in Lhasa in the tidal band also has low noise level, which contributes to the higher accuracy of the tidal parameters. Additionally, the tidal parameters obtained in this paper to a large extent match well with those given by the theoretical model.

Knowledge of the OSG-057 SG noise levels in Lhasa in different frequency bands provides a necessary precon- dition and reference for its applications to both global and regional research projects, such as the GGP/IGETS, APSG, and CMONOC.

Acknowledgements We are grateful to the GGP/IGETS and the SG stations for providing the SG data. We also thank the two Anonymous Reviewers for their valuable comments and suggestions. This work was jointly supported by the National Basic Research Program of China (Grant No. 2014CB845902), and the National Natural Science Foundation of China (Grant Nos. 41274085, 41474062, 41574072, 41374084).

\section{REFERENCES}

Amalvict, M., J. Hinderer, and S. Rózsa, 2006: Crustal vertical motion along a profile crossing the Rhine graben from the Vosges to the Black Forest Mountains: Results from absolute gravity, GPS and levelling observations. J. Geodyn., 41, 358-368, doi: 10.1016/j. jog.2005.08.036. [Link]

Baker, T. F. and M. S. Bos, 2003: Validating Earth and ocean tide models using tidal gravity measurements. Geophys. J. Int., 152, 468-485, doi: 10.1046/j.1365246X.2003.01863.x. [Link]

Banka, D., 1997: Noise levels of superconducting gravimeters at seismic frequencies. Ph.D. Thesis, GDMB - Informationgesellschaft mbH, Clausthal, Germany.

Banka, D. and D. Crossley, 1999: Noise levels of superconducting gravimeters at seismic frequencies. Geophys. J. Int., 139, 87-97, doi: 10.1046/j.1365246X.1999.00913.x. [Link]

Boy, J. P., M. Llubes, J. Hinderer, and N. Florsch, 2003: A comparison of tidal ocean loading models using superconducting gravimeter data. J. Geophys. Res., 108, doi: 10.1029/2002JB002050. [Link]

Braitenberg, C., 2014: Dynamic implications of temporal gravity changes over Himalaya-Tibet. $29^{\text {th }}$ HimalayaKarakoram-Tibet Workshop, Lucca, Italy, September 2-4, 2014.

Courtier, N., B. Ducarme, J. Goodkind, J. Hinderer, Y. Imanishi, N. Seama, H. Sun, J. Merriam, B. Bengert, and D. E. Smylie, 2000: Global superconducting gravimeter observations and the search for the translational modes of the inner core. Phys. Earth Planet. Inter., 117, 3-20, doi: 10.1016/S0031-9201(99)00083-7. [Link]

Crossley, D. and J. Hinderer, 2009: The contribution of GGP superconducting gravimeters to GGOS. In: Sideris, M. G. (Ed.), Observing our Changing Earth, Springer Berlin Heidelberg, 841-852, doi: 10.1007/978-3-54085426-5_97. [Link]

Crossley, D., J. Hinderer, G. Casula, O. Frnacis, H. T. Hsu, Y. Imanishi, G. Jentzsch, J. Kääriänen, J. Merriam, B. Meurers, J. Neumeyer, B. Richter, K. Shibuya, T. Sato, 
and T. van Dam, 1999: Network of superconducting gravimeters benefits a number of disciplines. Eos, Trans., AGU, 80, 121-126, doi: 10.1029/99EO00079. [Link]

Crossley, D., J. Hinderer, and J. P. Boy, 2005: Time variation of the European gravity field from superconducting gravimeters. Geophys. J. Int., 161, 257-264, doi: 10.1111/j.1365-246X.2005.02586.x. [Link]

Dehant, V., P. Defraigne, and J. M. Wahr, 1999: Tides for a convective Earth. J. Geophys. Res., 104, 1035-1058, doi: 10.1029/1998JB900051. [Link]

Ding, H. and B. F. Chao, 2015: The Slichter mode of the Earth: Revisit with optimal stacking and autoregressive methods on full superconducting gravimeter data set. J. Geophys. Res., 120, 7261-7272, doi: 10.1002/2015JB012203. [Link]

Ducarme, B. and L. Vandercoilden, 2000: First results of the GGP data bank at ICET. Bull. Inf. Marees Terr., 132, 10291-10298.

Dziewonski, A. M. and D. L. Anderson, 1981: Preliminary reference Earth model. Phys. Earth Planet. Inter., 25, 297-356, doi: 10.1016/0031-9201(81)90046-7. [Link]

Freybourger, M., J. Hinderer, and J. Trampert, 1997: Comparative study of superconducting gravimeters and broadband seismometers STS-1 / Z in seismic and subseismic frequency bands. Phys. Earth Planet. Inter., 101, 203217, doi: 10.1016/S0031-9201(97)00003-4. [Link]

Goodkind, J. M., 1991: The superconducting gravimeters principals of operation, current performance and future prospects. In: Poitevin, C. (Ed.), Proceedings of the Workshop on Non-Tidal Gravity Changes, Luxembourg, 81-90.

Hinderer, J. and D. Crossley, 2000: Time variations in gravity and inferences on the Earth's structure and dynamics. Surv. Geophys., 21, 1-45, doi: 10.1023/A:1006782528443. [Link]

Hinderer, J., D. Crossley, and O. Jensen, 1995: A search for the Slichter triplet in superconducting gravimeter data. Phys. Earth Planet. Inter., 90, 183-195, doi: 10.1016/0031-9201(95)05083-N. [Link]

Longuevergne, L., G. Ferhat, P. Ulrich, J. P. Boy, N. Florsch, and J. Hinderer, 2007: Towards physical modeling of local-scale hydrological contribution of soils for precise gravimetric corrections in Strasbourg. First Asia Workshop on Superconducting Gravimetry, Hsinchu, Taiwan.

Meurers, B., 2001: Superconducting gravimetry in geophysical research today. J. Geodetic Soc. Jap., 47, 300-307, doi: 10.11366/sokuchi1954.47.300. [Link]

Nawa, K., N. Suda, Y. Fukao, T. Sato, Y. Aoyama, and K. Shibuya, 1998: Incessant excitation of the Earth's free oscillations. Earth Planets Space, 50, 3-8, doi: 10.1186/BF03352080. [Link]

Nawa, K., N. Suda, Y. Fukao, T. Sato, Y. Tamura, K.
Shibuya, H. McQueen, H. Virtanen, and J. Kääriäinen, 2000: Incessant excitation of the Earth's free oscillations: Global comparison of superconducting gravimeter records. Phys. Earth Planet. Inter., 120, 289-297, doi: 10.1016/S0031-9201(00)00158-8. [Link]

Neumeyer, J., P. Schwintzer, F. Barthelmes, O. Dierks, Y. Imanishi, C. Kroner, B. Meurers, H. P. Sun, and H. Virtanen, 2005: Comparison of superconducting gravimeter and CHAMP satellite derived temporal gravity variations. In: Reigber, C., H. Lühr, P. Schwintzer, and J. Wickert (Eds.), Earth Observation with CHAMP: Results from Three Years in Orbit, Springer Berlin Heidelberg, 31-36, doi: 10.1007/3-540-26800-6_5. [Link]

Neumeyer, J., F. Barthelmes, O. Dierks, F. Flechtner, M. Harnisch, G. Harnisch, J. Hinderer, Y. Imanishi, C. Kroner, B. Meurers, S. Petrovic, Ch. Reigber, R. Schmidt, P. Schwintzer, H. P. Sun, and H. Virtanen, 2006: Combination of temporal gravity variations resulting from superconducting gravimeter $(\mathrm{SG})$ recordings, GRACE satellite observations and global hydrology models. J. Geodesy, 79, 573-585, doi: 10.1007/ s00190-005-0014-8. [Link]

Neumeyer, J., F. Barthelmes, C. Kroner, S. Petrovic, R. Schmidt, H. Virtanen, and H. Wilmes, 2008: Analysis of gravity field variations derived from Superconducting Gravimeter recordings, the GRACE satellite and hydrological models at selected European sites. Earth Planets Space, 60, 505-518, doi: 10.1186/BF03352817. [Link]

Nishida, K., N. Kobayashi, and Y. Fukao, 2002: Origin of earth's ground noise from 2 to $20 \mathrm{mHz}$. Geophys. Res. Lett., 29, doi: 10.1029/2001GL013862. [Link]

Okubo, S., S. Yoshida, T. Sato, Y. Tamura, and Y. Imanishi, 1997: Verifying the precision of a new generation absolute gravimeter FG5 - Comparison with superconducting gravimeters and detection of oceanic loading tide. Geophys. Res. Lett., 24, 489-492, doi: 10.1029/97GL00217. [Link]

Peterson, J., 1993: Observations and modeling of seismic background noise. Open-File Report 93-322, U.S. Department of Interior, Geological Survey, Albuquerque, New Mexico.

Richter, B., H. G. Wenzel, W. Zürn, and F. Klopping, 1995: From Chandler wobble to free oscillations: Comparison of cryogenic gravimeters and other instruments in a wide period range. Phys. Earth Planet. Inter., 91, 131-148, doi: 10.1016/0031-9201(95)03041-T. [Link]

Rosat, S. and J. Hinderer, 2011: Noise levels of superconducting gravimeters: Updated comparison and time stability. Bull. Seismol. Soc. Am., 101, 1233-1241, doi: 10.1785/0120100217. [Link]

Rosat, S., J. Hinderer, D. Crossley, and L. Rivera, 2003: The search for the Slichter mode: Comparison of noise lev- 
els of superconducting gravimeters and investigation of a stacking method. Phys. Earth Planet. Inter., 140, 183-202, doi: 10.1016/j.pepi.2003.07.010. [Link]

Rosat, S., J. Hinderer, D. Crossley, and J. P. Boy, 2004: Performance of superconducting gravimeters from longperiod seismology to tides. J. Geodyn., 38, 461-476, doi: 10.1016/j.jog.2004.07.005. [Link]

Smylie, D. E., 1992: The inner core translational triplet and the density near Earth's center. Science, 255, 16781682, doi: 10.1126/science.255.5052.1678. [Link]

Sun, H. and H. Xu, 1997: Execution and prospect for the Global Geodynamics Project cooperation. Adv. Earth Sci., 12, 152-157.

Sun, H., H. Xu, B. Ducarme, and J. Hinderer, 1999: Comprehensive comparison and analysis of the tidal gravity observations obtained with superconducting gravimeters at stations in China, Belgium and France. Chin. Sci. Bull., 44, 750-755, doi: 10.1007/BF02909719. [Link]

Sun, H. P., S. Takemoto, H. T. Hsu, T. Higashi, and A. Mukai, 2001: Precise tidal gravity recorded with superconducting gravimeters at stations Wuhan (China) and Kyoto (Japan). J. Geodesy, 74, 720-729, doi: 10.1007/ s001900000139. [Link]

Sun, W., Q. Wang, H. Li, Y. Wang, S. Okubo, D. Shao, D. Liu, and G. Fu, 2009: Gravity and GPS measurements reveal mass loss beneath the Tibetan Plateau: Geodetic evidence of increasing crustal thickness. Geophys. Res. Lett., 36, L02303, doi: 10.1029/2008GL036512.
[Link]

Sun, W., Q. Wang, H. Li, Y. Wang, and S. Okubo, 2011: A reinvestigation of crustal thickness in the Tibetan Plateau using absolute gravity, GPS and GRACE data. Terr. Atmos. Ocean. Sci., 22, 109-119, doi: 10.3319/ TAO.2010.06.07.01(TibXS). [Link]

Tamura, Y., 1987: A harmonic development of the tidegenerating potential. Bull. Inf. Marées Terrestres, 99, 6813-6855.

Van Camp, M. and P. Vauterin, 2005: Tsoft: Graphical and interactive software for the analysis of time series and Earth tides. Comput. Geosci., 31, 631-640, doi: 10.1016/j.cageo.2004.11.015. [Link]

Wenzel, H. G., 1996: The nanogal software: Earth tide data processing package ETERNA 3.30. Bull. Inf. Marées Terrestres, 124, 9425-9439.

Xi, Q., 1989: A new complete development of the tidegenerating potential for the epoch J2000.0. Bull. Inf. Marées Terrestres, 99, 6766-6812.

Xu, J., X. Chen, J. Zhou, and H. Sun, 2012: Characteristics of tidal gravity changes in Lhasa, Tibet, China. Chin. Sci. Bull., 57, 2586-2594, doi: 10.1007/s11434-0125130-2. [Link]

Zhang, M., J. Xu, H. Sun, W. Shen, and X. Chen, 2014: Comparison of noise levels of the new $i \mathrm{Grav}-007$ superconducting gravimeter and the SG-065 superconducting gravimeter in Wuhan (China). Bull. Inf. Marées Terrestres, 148, 11987-12000. 